\title{
Study and Numerical Simulation on Explosion Propagation Characteristics of Lignite Dust
}

\author{
Zizheng Pang ${ }^{1, \mathrm{a}}$, Shunbing $\mathrm{Zhu}^{2, \mathrm{~b}^{*}}$, and Yanru $\mathrm{He}^{1}$ \\ ${ }^{1}$ College of Safety Science and Engineering, Nanjing Tech University, China \\ ${ }^{2}$ College of Safety Science and Engineering, Nanjing Tech University, China
}

\begin{abstract}
In order to reveal the explosion propagation law of lignite dust in $20 \mathrm{~L}$ spherical explosion test device, the dust diffusion behavior and explosion propagation characteristics of lignite were studied by experiment and numerical simulation. The propagation process of dust explosion is studied by using high-speed camera and 20L spherical explosion test system, and the process of dust diffusion and explosion is simulated by using FLUENT software. The results show that the explosion propagation of lignite dust in the 20L spherical explosion test system has four different stages: the first explosion stage, the full explosion combustion stage, the continuous combustion stage and the combustion attenuation stage. The test results are slightly different from that of the fluent simulation of lignite dust explosion by using the high-speed camera to collect the dust explosion images. Results within the allowable error range, the experimental image of explosion combustion of lignite dust is well connected with the simulation results, which has a good display effect on the explosion propagation of lignite dust.
\end{abstract}

\section{Introduce}

With industrial development, combustible dust explosion accidents occur frequently, dust explosions very destructive, causing serious casualties and economic losses of property. Among then, coal dust explosion has always been one of the objects studied by scholars.

In the study of explosion propagation characteristics, Bing $\mathrm{Du}^{[1]}$ et al. studied that dust explosions are closely related to the dispersion behavior of dust, using high-speed cameras to record the dispersion process of typical carbonaceous dust, and using image processing technology for qualitative analysis; Xueling $\mathrm{Liu}^{[2]}$ et al. used their own designed 20L cylindrical transparent plexiglass, cast iron explosion test tank and symmetrical double nozzle pneumatic powder spraying and dispersing device to obtain the explosive characteristic parameters of coal powder under different experimental conditions and gave a quantitative analysis; Weiguo $\mathrm{Cao}^{[3]}$ et al. used high-speed cameras and infrared thermal imaging devices to volatile matter of two different mass fractions Study the flame propagation process and the temperature distribution in space of the coal powder.Mercer and
Dahoe ${ }^{[4,5]}$ used three different types of nozzles for comparative experiments, and discussed the experimental research results of turbulent attenuation in a $20 \mathrm{~L}$ closed ball container.

Most of the flame propagation research is now concentrated in cylindrical explosion containers,and there is little research on dust explosion in space. Therefore,this article has studied in detail the flame propagation characteristics of lignite dust in a $20 \mathrm{~L}$ spherical explosion container,and Starting with the nature of the explosion,an experimental simulation and comparative study on the flame propagation of lignite dust was carried out.

\section{Experiment}

The experiment was conducted using a $20 \mathrm{~L}$ spherical explosion test system and a high-speed camera ${ }^{[6]}$. The installation location of the $20 \mathrm{~L}$ spherical explosion test system and high-speed camera is shown in fig.1.The lignite sample used in the experiment was ground, screened and dried. Characterize the particles with a laser particle size analyzer as shown in fig. 2 . 


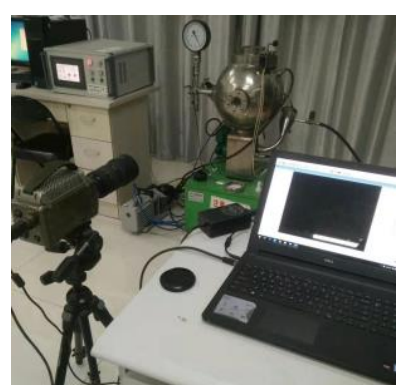

Fig.1. Experimental environment

\section{Numerical simulation}

\subsection{Establishment of geometric model}

Using the pre-processing software ICEM CFD, the 20L exploding spherical test system was simplified and the geometric point, line and plane models were constructed as shown in fig. 3 .

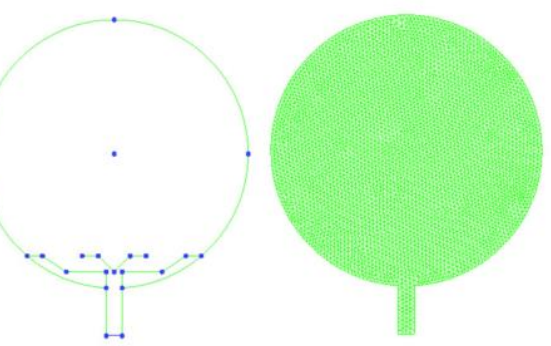

Fig.3. Geometric model and mesh generation

\subsection{Governing equation and calculation model}

Based on chemical reaction dynamics and fluid mechanics, the main equations are as follows:

Mass conservation equation:

$$
\frac{\partial \rho}{\partial \mathrm{t}}+\frac{\partial}{\partial x_{i}}\left(\rho u_{i}\right)=S_{m}
$$

Under the inertial coordinates, the momentum conservation equation $^{[7]}$ of direction I is:

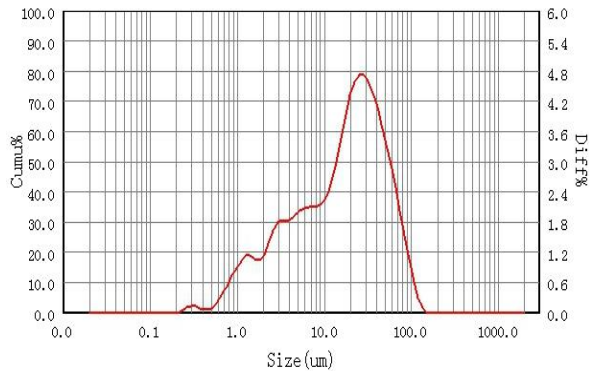

Fig.2. Particle size analysis of pulverized coal

$$
\frac{\partial}{\partial \mathrm{t}}\left(\rho u_{i}\right)+\frac{\partial}{\partial \mathrm{x}_{\mathrm{j}}}\left(\rho u_{i} u_{j}\right)=-\frac{\partial p}{\partial x_{i}}+\frac{\partial \tau_{i j}}{\partial c_{j}}+\rho g_{i}+F_{i}
$$

In the equation, $\mathrm{p}$ is the static pressure, ${ }^{\rho \mathrm{g}_{\mathrm{j}}}$ and $F_{\mathrm{j}}$ is the neutral volume force and other volume forces, $F_{\mathrm{j}}$ as well as other model source terms or custom source terms, and $\tau_{\mathrm{ij}}$ is the stress tensor.

For the calculation model selection of coal sample:the two-phase flow model uses the discrete phase model, the turbulence model uses the $\mathrm{K}-\varepsilon$ model, the combustion model selects the single rate model, the radiation model uses the DO model, and the gas phase turbulent combustion model uses the EBU-Arrhenius model.

\section{Results and discussion}

\subsection{Study on flame propagation process}

Using high speed camera within 20L spherical explosion test system of dust clouds explosion propagation.The images showed that there were two explosions during the experiment. The first explosion was caused by the explosion of the chemical ignition head, as shown in fig. 4. The duration of the explosion was about $18 \mathrm{~ms}$.

The second explosion was a coal dust explosion, and the second explosion followed the first explosion, as shown in fig.5. The entire combustion process is divided into the following four stages, each of which has a different form of explosion.

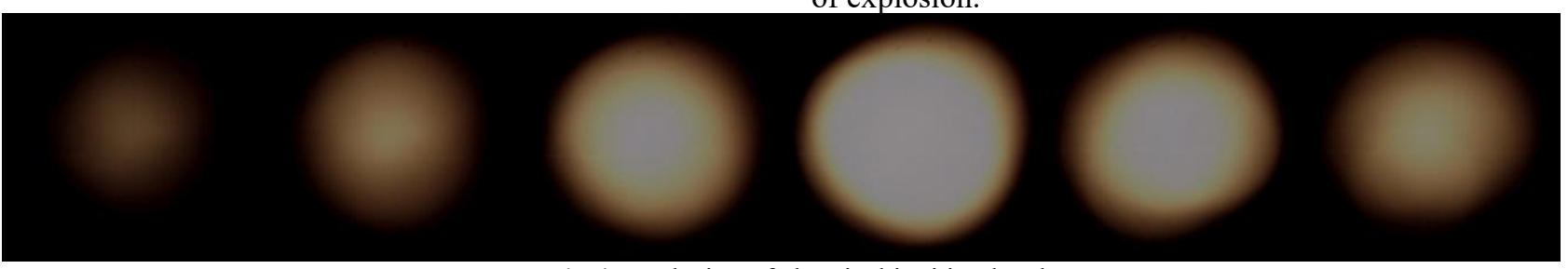

Fig.4. Explosion of chemical ignition head

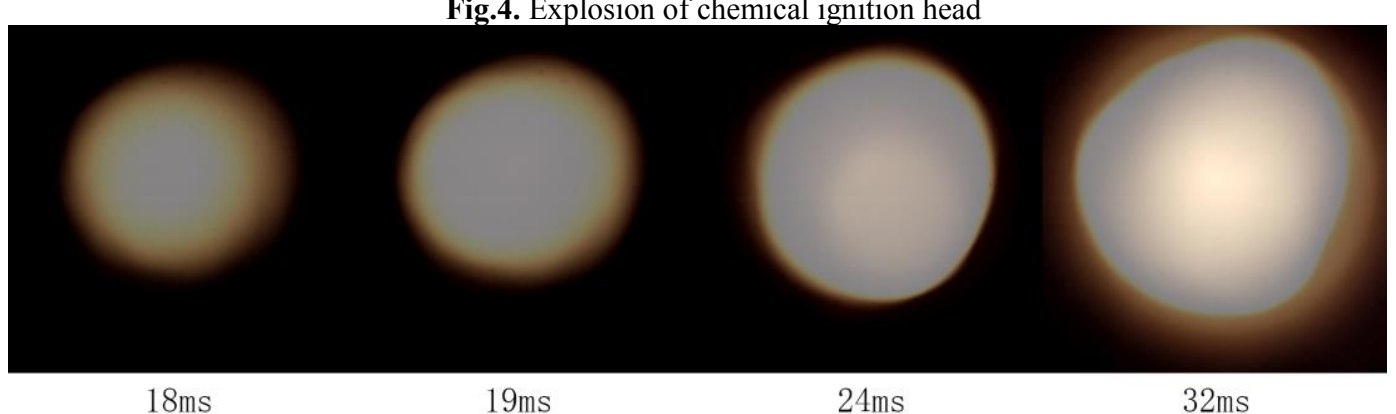

Fig.5. First stage explosion 
The first stage is the initial explosion of dust into the explosion tank, and the duration of this stage is about $14 \mathrm{~ms}$. After the dust enters the 20L spherical explosion container, it encounters the heat generated by the ignition head and is ignited when the dust distribution is not even, resulting in the first uneven explosion, as shown in fig.5. It can be seen in fig. 8 that after the unevenly distributed dust is ignited, a dim fireball appears. As the dust distribution begins to become uniform, the fireball produced by the explosion begins to become larger, and the center of the fireball appears higher than the surrounding strong light spot, and the strong light spot As the fireball becomes larger, the relative strain becomes larger.

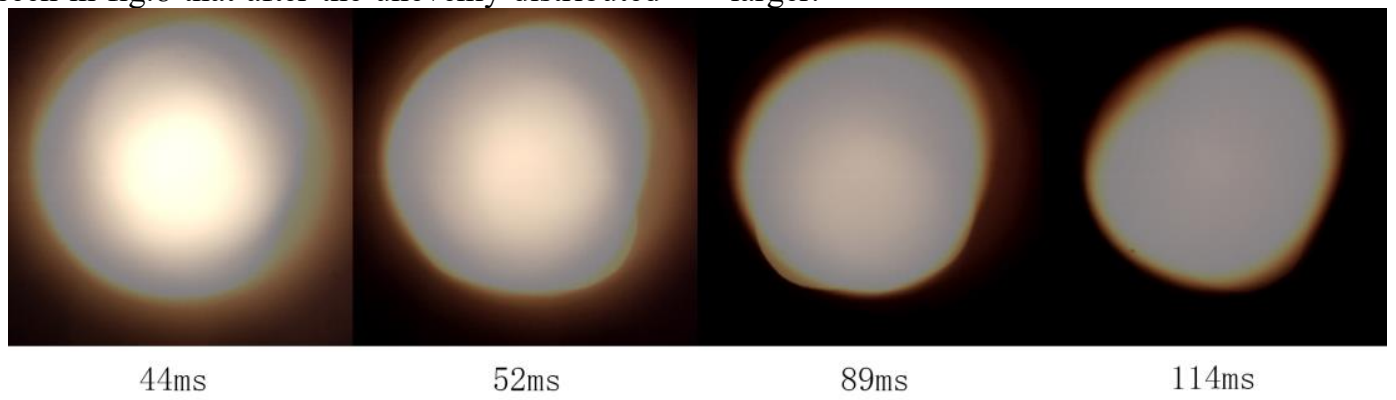

Fig.6. Second stage explosion

The second stage is the full combustion explosion of the dust in the 20L spherical explosion vessel. The duration of this stage is about $70 \mathrm{~ms}$. As shown in fig.6, as the dust spreads more evenly, the explosion becomes more intense and the central strong light spot becomes brighter. After that, the central strong light spot gradually became smaller, but the convex outer flame continued to be generated, but as the combustible dust in the $20 \mathrm{~L}$ spherical explosion vessel decreased, the explosion reaction began to slow down.
The third stage is the continuous combustion and explosion of dust, and the duration of this stage is about $51 \mathrm{~ms}$. As shown in fig.7, after the violent explosion of brown coal dust, the remaining dust is reduced, but the heat generated in the second stage of explosion can still maintain the basic combustion of the dust, the strong light part at the center of the fireball begins to completely disappear, and the two lights at the center of the fireball gradually decrease.

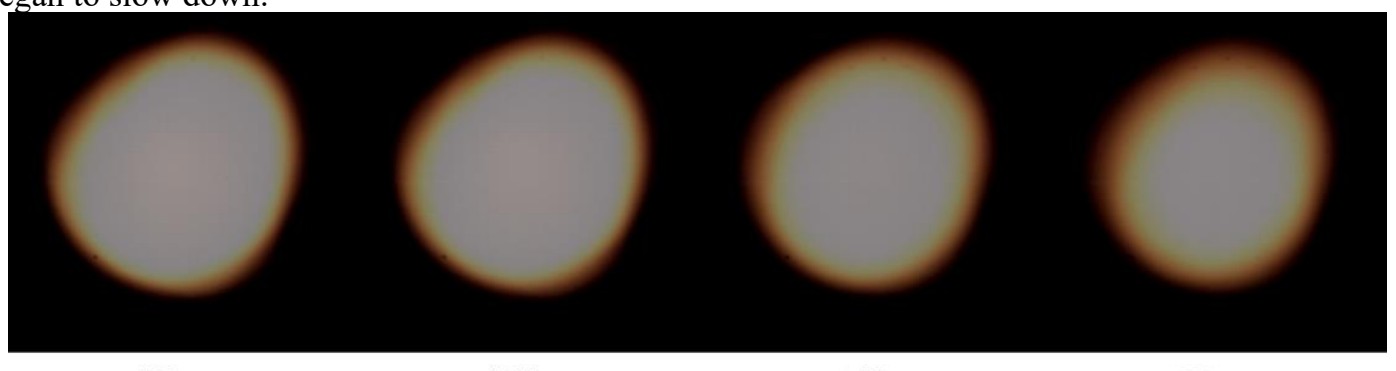

$123 \mathrm{~ms}$

$125 \mathrm{~ms}$

$147 \mathrm{~ms}$

$174 \mathrm{~ms}$

Fig.7. Third stage explosion

The fourth stage is the decay combustion stage of the dust. The duration of the whole process is about $103 \mathrm{~ms}$. As shown in fig.8, due to the sudden decrease of the dust content in the explosion container, the dust cloud burns slowly, and the central bright part basically disappears. The outer dark yellow area gradually extended to the center, the shape and size of the entire fireball also continued to decrease, and finally the explosion ended.
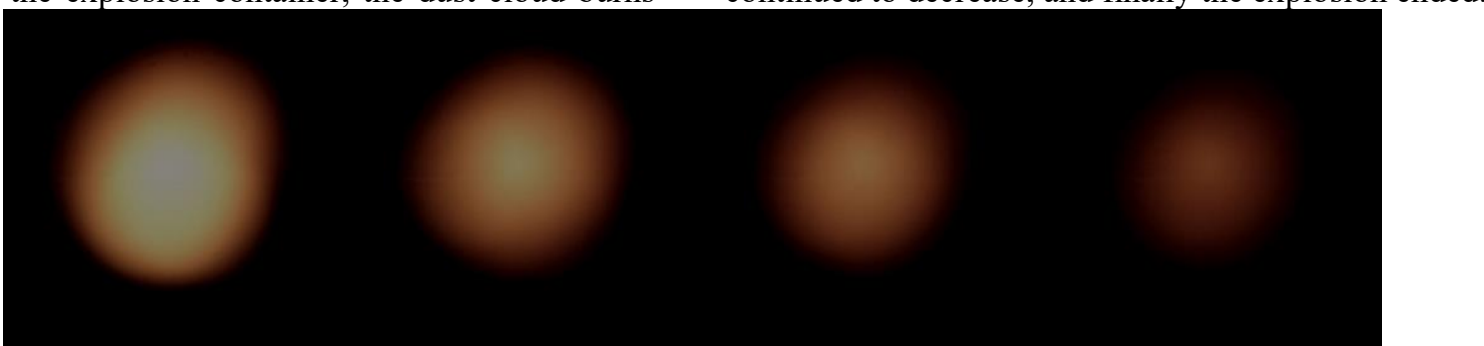

$$
230 \mathrm{~ms}
$$

$264 \mathrm{~ms}$

$292 \mathrm{~ms}$

$333 \mathrm{~ms}$

Fig.8. Fourth stage explosion

After the chemical ignition head is ignited, the following combustion phenomena occur:as shown in fig.9, when the lignite dust is ignited for $34 \mathrm{~ms}$, the explosion cloud begins to explode locally, and the whole explosion process lasts for a period of time. After lignite is ignited, with the increase of temperature, dust particles are gasified under the action of high temperature, and too much gas is produced, resulting in the combustion of gas like a drum. The whole phenomenon lasted for $73 \mathrm{~ms}$, and there was no significant change in the shape of fireball. 


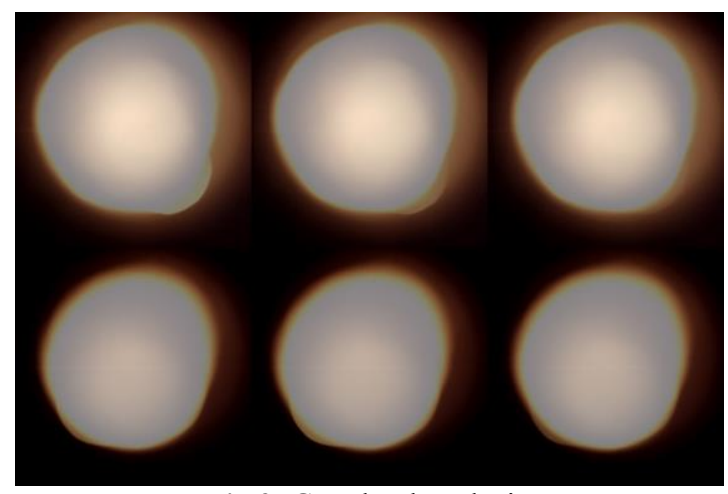

Fig.9. Gas cloud explosion

\subsection{Comparison of flame propagation and explosion process}

Fig.10 shown the real-time data diagram of explosion pressure and explosion intensity obtained by $20 \mathrm{~L}$ spherical explosion test system. From the figure, we can

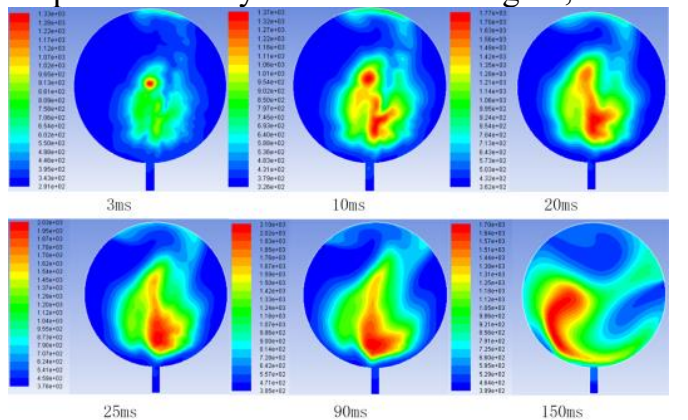

Fig.11. Simulation results of dust explosion at different times

\subsection{Comparison of flame propagation experiment and simulation}

After chemical igniter ignites pyrolysis volatile component began, the high temperature region of $1330 \mathrm{~K}$, and the high-temperature region is small, as the combustion proceeds, the highest temperature reached after $20 \mathrm{~ms} 1770 \mathrm{~K}$, and has not been ignited region increased from $300 \mathrm{~K}$ to $362 \mathrm{~K}$. $90 \mathrm{~ms}$ after ignition, the temperature reached a maximum value, when the temperature reaches the high temperature region of $2100 \mathrm{~K}$, is not ignited region increased from $300 \mathrm{~K}$ to $385 \mathrm{~K}$, then coal dust in the container substantially complete combustion, the temperature in the vessel began to fall, at $150 \mathrm{~ms}$, the maximum temperature lowered to $1700 \mathrm{~K}$, but the combustion heat to the surroundings so that the diffusion zone temperature is still rising unburnt and reaches $399 \mathrm{~K}$.

Through the comparison between fig. 11 and fig.12, it is found that the two pictures can better restore the whole process of dust explosion, the sharp brightness changes exhibited by the image from $10 \mathrm{~ms}$ to $25 \mathrm{~ms}$ can be well represented as temperature changes in the simulation.It can be seen that the simulation results of fluent basically conform to the experimental phenomenon.

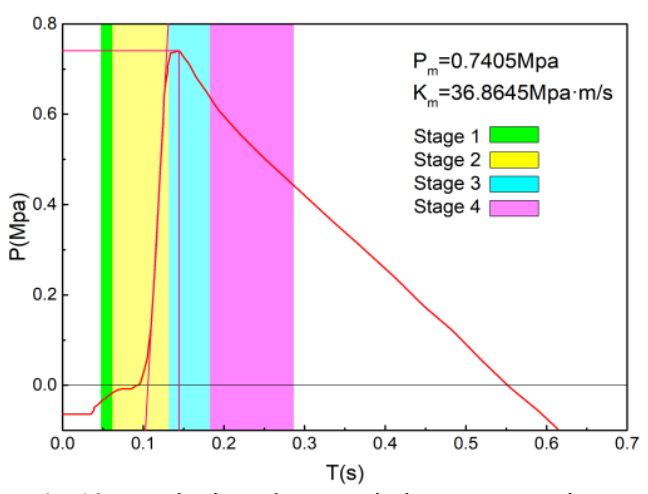

Fig.10. Explosion characteristic parameter image see that the maximum explosion pressure occurs about $120 \mathrm{~ms}$ after air inlet ignition, and the maximum explosion intensity occurs about $90 \mathrm{~ms}$ after air inlet ignition. According to the above steps, the dust explosion phenomenon in the second stage is consistent.
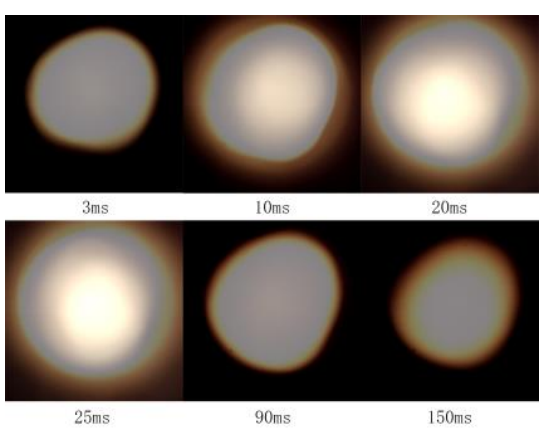

Fig.12. Experimental results at different time

\section{Conclusion}

The purpose of this study is to have a general understanding of the combustion and explosion propagation characteristics of lignite dust. A high-speed camera was used to record the dust explosion in a $20 \mathrm{~L}$ spherical explosion vessel, and the numerical simulation method was used to compare with the experimental results. The results are as follows:

1.The lignite dust explosion is divided into four stages, the initial explosion stage, the full explosion combustion stage, the continuous combustion stage, and the decay combustion stage. Among them, the chemical changes involved in the second stage are more obvious. Combustible gas combustion caused by severe explosion. 2.The gasification explosion reaction in the second stage of lignite dust combustion explosion is more violent. A large amount of gas is generated during the combustion process, and the gas rushes out of the fireball to produce a partial gas cloud explosion; the entire explosion process basically corresponds to the change of the entire explosion curve During the process, the time range of the explosion intensity coincides with the second stage of the explosion. 
3.The simulation results reflect the temperature performance of the brown coal dust explosion and can well reflect the explosion of the brown coal dust in the 20L spherical explosion container. The dust explosion patterns at each time period are basically consistent with the simulated images.

\section{References}

1. Bing Du,Weixing Huang,Long Liu,Tan Zhang,Hao Li,Yidan Ren,Hanlin Wang. Visualization and analysis of dispersion process of combustible dust in a transparent Siwek 20-L chamber[J]. Journal of Loss Prevention in the Process Industries,2015,33.

2. Xueling Liu,Qi Zhang.Influence of turbulence flow on explosion characteristics of coal dust in 20L vessel[J].Journal of China Coal Society,2018,43(11):3137-3144.

3. Tianqi Liu.Study on flame heat release model of different quality coal dust explosion and case [J].Fire science and technology,2019,38(12):1676-1680.

4. David B Mercer,Paul R Amyotte,Debbie J Dupuis, Michael J Pegg,Arief Dahoe, Wouter B.C de Heij,John F Zevenbergen,Brian Scarlett. The influence of injector design on the decay of pre-ignition turbulence in a spherical explosion chamber[J]. Journal of Loss Prevention in the Process Industries,2001,14(4).

5. A.E. Dahoe,R.S. Cant,M.J. Pegg,B. Scarlett. On the transient flow in the 20-liter explosion sphere[J]. Journal of Loss Prevention in the Process Industries,2001,14(6).

6. Li Haitao,Chen Xiaokun,Deng Jun,Shu Chi-Min,Kuo Chia-Ho, Yu Yongchuan,Hu Xiangyu. CFD analysis and experimental study on the effect of oxygen level, particle size, and dust concentration on the flame evolution characteristics and explosion severity of cornstarch dust cloud deflagration in a spherical chamber[J]. Powder Technology,2020(prepublish).

7. Chaoguang Zhang,Juncheng Jiang,Zhiqin Zheng.Study on the Mode and Prevention of Dust Explosion Accident[J].China Safety Science Journal,2005(06):73-76+115. 